\title{
Curvas granulométricas como alternativa para la caracterización del material rocoso en minería
}

\author{
Feijoo Patricio ${ }^{1}$ \\ ORCID: https://orcid.org/0000-0001-6901-7933 \\ pfeijoo@uazuay.edu.ec \\ Universidad del Azuay \\ Cuenca-Ecuador
}

\author{
Bustamante Esteban ${ }^{2}$ \\ ORCID: https://orcid.org/0000-0002-1200-9681 \\ estebanbus@es.uazuay.edu.ec. \\ Universidad del Azuay \\ Cuenca-Ecuador
}

\author{
Guillén Christian ${ }^{3}$ \\ ORCID: https://orcid.org/0000-0003-2950-6067 \\ cg8uazuay.edu.ec@es.uazuay.edu.ec \\ Universidad del Azuay \\ Cuenca-Ecuador
}

Recibido (08/02/21), Aceptado (18/02/21)

Resumen: En proyectos mineros es importante determinar ciertos parámetros, como la resistencia a compresión simple (RCS) del material rocoso, ya que es fundamental para el uso de clasificaciones geomecánicas, como RMR o Índice Q, las que establecen geometrías para estabilidad en canteras y fortificación en minas subterráneas, evitando desprendimientos de rocas o derrumbes, garantizando la salud de trabajadores y protección de equipos. Pero, para conocer la RCS, es necesario enviar muestras al laboratorio, lo cual hace tediosa y costosa su valoración. Este trabajo introduce una propuesta para la determinación de este parámetro, mediante una sucesión simple y sencilla de procesos mecánicos. Obtenidas muestras de roca, prepararlas, triturarlas, clasificarlas y valorada su RCS, se establece una relación entre estos parámetros, evitando enviar permanentemente muestras al laboratorio. Esta relación se presenta de una manera gráfica, de fácil interpretación y propone una ecuación, para de esta forma obtener el parámetro de una manera aproximada.

Palabra Claves: Minería, trituración de rocas, curvas granulométricas, resistencia a la compresión uniaxial.

\section{Granulometric curves as an alternative for the characterization of rocky material in mining}

\begin{abstract}
In mining projects, is important to determine certain parameters, such as the unconfined compressive strength (UCS) of the rock material, since it is fundamental for the use of geomechanical classifications, such as RMR or Q Index, which establish geometries for stability in quarries and fortification in underground mines, avoiding rock falls or landslides, guaranteeing the health of workers and protecting equipment. But, to know the UCS, it is necessary to send samples to the laboratory, which makes its evaluation tedious and expensive. This work introduces a proposal for the determination of this parameter, through a simple and straightforward sequence of mechanical processes. Once rock samples are obtained, prepare them, crush them, classify them and assess their UCS, a relationship is established between these parameters, avoiding permanently sending samples to the laboratory. This relationship is presented in a graphical way, easy to interpret and proposes an equation, in order to obtain the parameter in an approximate way.
\end{abstract}

Keywords: Mining, rock crushing, granulometric curves, uniaxial compressive strength.

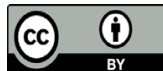




\section{I.INTRODUCCIÓN}

En minería se cuentan con equipos básicos para la ejecución de la explotación de minerales o materiales pétreos, debido a esto y a la ubicación de los depósitos, en muchas ocasiones se dificulta el hecho de enviar permanentemente muestras al laboratorio para obtener la resistencia a la compresión simple del material rocoso, por lo que los técnicos o ingenieros en minas estamos en la búsqueda constante de ensayos in situ y así poder establecer una solución al problema.

En pequeña minería, específicamente, la determinación de este parámetro es un problema permanente, ya que la toma de muestras del material rocoso conlleva un tiempo y costo que dificulta a las empresas y muchas de las ocasiones se lo deja de realizar generando una falta de análisis de las estructuras mineras en términos de seguridad.

La caracterización del material rocoso es fundamental para la evaluación de las condiciones de estabilidad que presentan los macizos rocosos, por lo que este parámetro se lo debe obtener indispensablemente. Pero muchas veces debido a las condiciones en las cuales se encuentran las labores mineras, resulta complicado y a veces casi imposible enviar muestras de roca a laboratorios para determinar el valor de la RCS, y en estos tiempos es la problemática de los encargados de evaluar la estabilidad de las estructuras en el campo, por lo que se presenta la imperiosa necesidad de generar alternativas in situ para la valoración de la RCS 1.

Por esta razón se propone una metodología que correlaciona la resistencia a la compresión simple de la roca estudiada y su granulometría, caracterizando el material rocoso. Los resultados son alentadores y coadyuvan a determinar la propiedad mencionada, con una aproximación aceptable para efectos de los cálculos posteriores.

\section{II.DESARROLLO}

El comportamiento mecánico de una masa de roca in situ es diferente de una masa de suelo. Los dos motivos fundamentales que actúan juntos son los siguientes: a) El material rocoso es mucho más fuerte y resistente que el material de suelo. b) Una masa rocosa presenta casi siempre sistemas de debilidades estructurales (fracturas, diaclasas, fisuras, juntas, discontinuidades, fallas de varios tamaños).

Por este motivo se tiene que distinguir entre macizo rocoso y material rocoso. Se habla de material rocoso entendiendo el material intacto, es decir un testigo o muestra de pequeño tamaño generalmente constituido por material sin fracturas. Se habla de macizo rocoso a todo el conjunto del material rocoso y de las discontinuidades presentes en él. Un macizo rocoso es por esto constituido por muchos bloques de material rocoso separados por discontinuidades, fisuras, etc. 2 .

En la caracterización del macizo rocoso, a partir de afloramientos, se realizan una serie de observaciones y medidas de campo que son la base y sistemática para que quede caracterizado. Hay que tener en cuenta, el número y orientación de las familias, tamaño del bloque e intensidad de la fracturación, grado de meteorización y resistencia del macizo 3.

En muchos proyectos de ingeniería de minas, la clasificación de los macizos rocosos se realiza inicialmente para determinar la estabilidad de las obras a ejecutar. Estas clasificaciones son conocidas como clasificaciones geomecánicas y derivan de la diferencia existente entre las propiedades de la roca por su naturaleza y las del macizo, que presentan diferentes grados de fracturación y meteorización. Las clasificaciones geomecánicas son sistemas de valoración del comportamiento del terreno rocoso. Se basan en calificar numéricamente las propiedades y características específicas de la roca en un emplazamiento determinado y posteriormente obtener una calificación final como la suma de las valoraciones parciales 4. Las clasificaciones geomecánicas modernas más difundidas en la ingeniería de túneles son las conocidas como Rock Mass Rating o sistema RMR, la clasificación del Instituto Geotécnico Noruego, o sistema Q y el Geological Strength Index (GSI). Las primeras dos clasificaciones se basan en los datos de recuperación de núcleos (RQD), número de familias de discontinuidades, rugosidad y estado general de las juntas, presencia de agua y adicionalmente pueden considerar la resistencia de la roca matriz, la orientación de las discontinuidades respecto a la excavación y el tipo de obra de que se trate 5 .

Una de las propiedades importantes que se debe conocer sobre el material rocoso es la resistencia a la compresión simple o uniaxial (RCS), y para obtener este parámetro se deben obtener muestras de roca que aflore en el yacimiento, preparar probetas adecuadas y enviarlas a laboratorios para la determinación de la presión de ruptura. Este trabajo debe ser permanente ya que la geología de los proyectos mineros varía en el avance de la explotación y por naturaleza las rocas son anisótropas y heterogéneas 6. 


\section{A.Resistencia a la Compresión Uniaxial (RCU).}

El comportamiento de un macizo rocoso es diferente de un material rocoso, debido a que el material rocoso es más fuerte y un macizo rocoso presenta casi siempre sistemas de debilidades estructurales llamadas juntas. Se habla de material rocoso cuando el material es intacto, por ejemplo, un testigo o muestra de pequeño tamaño que no presenta fracturas. Se habla de macizo rocoso a todo el conjunto de material rocoso y discontinuidades que lo conforman. En cuanto a la matriz rocosa, material rocoso o simplemente roca, hay muchos parámetros que se emplean para su identificación y descripción de sus características. Estas propiedades junto con la composición mineralógica determinan su comportamiento. La matriz rocosa rompe a favor de las superficies de fractura que se generan al superarse su resistencia pico. Así, de una forma indirecta los ensayos de compresión miden la resistencia al corte de las rocas. La resistencia a la compresión uniaxial de la matriz rocosa es un parámetro muy variable que depende de muchos factores. Factores intrínsecos: tipo de roca (factor condicionante básico), grado de microfisuración, porosidad y grado de alteración y/o meteorización. Esto da lugar a que no solo varíe la resistencia de un tipo de roca a otra, sino que, dentro de un mismo tipo de roca, se pueda dar un amplio rango de valores. Esta dispersión de valores, se corresponde a valores "medidos" de la resistencia, que están también influidos por. Factores extrínsecos: características del ensayo y características de la muestra.

La resistencia a la compresión simple ha sido uno de los parámetros clasificatorios de las rocas desde el punto de vista ingenieril 7. La resistencia a la compresión simple de las rocas también es muy importante cuando se van a utilizar métodos de arranque de materiales mediante el uso de explosivos. Las rocas responden de forma muy diferente a la onda de detonación del explosivo en lo que respecta a la primera etapa de formación de fisuras, primordial y origen del fenómeno de rotura y fragmentación.

Las rocas extremadamente duras pero frágiles rompen bien mientras que las rocas blandas elásticas apenas son fragmentadas 8. De igual forma es importante para el cálculo del uso de explosivos en métodos como el propuesto por López-Jimeno para los respectivos parámetros de la voladura 9. La resistencia de una roca o de sedimentos no bien consolidados está influida por la mineralogía de sus partículas y por el contacto que hay entre ellas 10.

Se suele entender por "resistencia" a la carga específica (por unidad de sección), que es necesario aplicar a un material para producir su rotura. Según el esfuerzo que se aplique la resistencia puede ser: a compresión, a tracción, a flexión, a corte, a torsión, etc. La resistencia a la compresión simple (o compresión uniaxial) de la matriz rocosa, es el valor que se obtiene cuando se aplica una carga de compresión en una dirección dada, sin aplicar ningún otro esfuerzo en ninguna otra dirección.

Se suele expresar con la siguiente relación:

$$
R C U=\frac{P}{A}
$$

Donde:

P es la carga de rotura y A es la sección sobre la que se aplica.

A continuación, podemos observar en la tabla 1, la clasificación de las rocas según la resistencia a la compresión. 
Tabla 1. Clasificación de las rocas en función de la Resistencia a Compresión Simple (ISRM)

\begin{tabular}{|c|c|}
\hline Descripción & $\begin{array}{c}\text { Resistencia a la Compresión Simple o } \\
\text { Uniaxial } \\
\text { (MPa) }\end{array}$ \\
\hline Resistencia muy baja & 1 al 25 \\
\hline Resistencia baja & $25-50$ \\
\hline Resistencia media & $50-100$ \\
\hline Resistencia alta & $100-200$ \\
\hline Resistencia muy alta & $>200$ \\
\hline
\end{tabular}

\section{B.Trituración de Rocas.}

Para hablar del proceso de trituración necesitamos entender sobre los tipos de máquinas que existen para esta actividad, industrialmente se utilizan diferentes tipos de máquinas de trituración. Se clasifican de acuerdo a la etapa y al tamaño de material tratado.

Trituradoras Primarias. Fragmentan trozos grandes hasta un producto de 8" a 6". Se tienen dos tipos de máquinas: Trituradoras de Mandíbulas y Trituradoras Giratorias.

Trituradoras Secundarias. Fragmentan el producto de la trituración primaria hasta tamaños de 3" a 2" entre estas máquinas tenemos: Trituradoras Giratorias, Trituradoras Cónicas y Trituradoras Hydrocónicas.

Trituradoras Terciarias. Fragmentan el producto de la trituración secundaria hasta tamaños de 1/2" o 3/8", entre estas máquinas tenemos: Trituradoras Cónicas y Trituradoras de Rodillos 11.

En adelante desarrollaremos la información pertinente a este tema. Los equipos más comunes que podemos encontrar en el medio son las trituradoras de mandíbulas, las de cono, de martillos, de impacto (vertical y horizontal), y de rodillos. Estas trituradoras se pueden clasificar en dos grandes grupos: Las trituradoras por compresión y las de impacto 12.

Trituradoras por compresión.

Son aquellas máquinas que comprimen mecánicamente las rocas dentro de una cámara hasta que se fracture la roca en partículas menores. Las trituradoras de mandíbulas, cono y de rodillos operan según el principio de compresión.

\section{Trituradora de mandíbula (Jaw crusher).}

Las trituradoras de mandíbulas se usan principalmente en la etapa primaria. La fracturación de las rocas ocurre en la cámara de trituración, la que está compuesta de una mandíbula fija y una mandíbula móvil. Estas mandíbulas están provistas de unas placas dentadas de acero al manganeso llamadas forros. El forro fijo va instalado a la mandíbula que no tiene movimiento y es ésta la que recibe la presión, el forro móvil está montado en la mandíbula que tiene el movimiento oscilante por acción de un eje excéntrico, estos forros deben reemplazarse regularmente, debido al desgaste que se genera por la fricción con las rocas. Hay dos tipos básicos de trituradoras de mandíbulas, las de un solo efecto y las de doble efecto. La trituradora de un solo efecto cuenta con un eje excéntrico en la parte superior de la estructura. La rotación del eje junto con la placa basculante produce una acción lenta de vaivén con mucha energía. Una trituradora de doble efecto tiene básicamente dos ejes y dos placas basculantes. El primer eje es un eje pivotante en la parte superior de la trituradora, mientras que el otro es un eje excéntrico que acciona las dos placas articuladas. La mandíbula móvil tiene un movimiento puro de vaivén hacia la mandíbula fija. El movimiento oscilante que causa compresión, tanto en la entrada como en la descarga de material, le da a la trituradora de simple efecto una mejor capacidad que de las trituradoras de doble efecto de tamaño similar. La trituradora de mandíbulas es un equipo robusto y fiable, y por ello muy popular en plantas de trituración primaria. La capacidad de producción de la trituradora depende de las características de las materias a triturar (intensidad, dureza, y composición de granulosidad alimentada, etc.), las filtraciones de la trituradora y las condiciones de operación (casos de alimentación y dimensión de la salida de materias), etc. 13. 


\section{C.Clasificación Granulométrica.}

La clasificación o cribado es el proceso donde se separan las fracciones de roca. El análisis granulométrico es una prueba en donde se determina la distribución de partículas de diferentes tamaños por medio de cribas 14. Esta selección se hace a través de mallas metálicas o poliuretano que van montadas en las cribas vibratorias. Los principios de cribado para cualquier tipo de material en zarandas vibratorias son básicamente los mismos, los resultados dependerán mucho de las configuraciones que se hayan aplicado. Una vez que el material esté sobre la superficie de la caja de cribado, se genera el fenómeno de la estratificación, en cuyo proceso por efecto del movimiento vibratorio, las partículas gruesas emergen a la parte superior de la capa de material y las partículas más pequeñas buscan su camino hacia la parte inferior de la capa a través de los espacios creados entre las partículas gruesas. Los factores que afectan la estratificación son: Velocidad del flujo de material, el espesor de la capa del material, características de inclinación de la criba, características de la potencia del motor, configuración de la amplitud, dirección de rotación del elemento excéntrico, tipo de movimiento y frecuencia y humedad superficial de las partículas, un alto contenido de humedad dificulta la estratificación con poca probabilidad de separación. Grado de limpieza con referencia a la plasticidad.

Por este procedimiento, el tamaño de partícula se asocia al número de aberturas que tiene el tamiz por pulgada lineal, utilizando el concepto de tamaño de FERET que se define como el tamaño que corresponde a la distancia entre dos tangentes paralelas a la partícula, trazadas en la misma dirección de la medición 15.

El análisis granulométrico o distribución de partículas del material tratado, es importante en los procesos de investigación para un control de la operación. El análisis granulométrico (o análisis del tamaño de partículas) del material tratado, cuyo resultado puede ser representado en forma de tabla o por medio de la respectiva curva granulométrica, reviste una gran importancia para cualquier proceso de tratamiento, sea en fase de proyecto y estudio en laboratorio, sea para el control de las operaciones durante el funcionamiento de una planta. El análisis granulométrico es generalmente llevado a cabo con métodos mecánicos (serie de cribas o tamices) o con métodos hidráulicos y neumáticos. En general las cribas se usan para clasificaciones hasta tamaños superiores a 40 micrones indicativamente, mientras que las clasificaciones hidráulica y neumática son utilizadas para clasificar a tamaños menores. Para clasificación a tamaños menores que 40 micrones se está desarrollando también el nuevo campo de las técnicas electrónicas 16.

La granulometría, tema que nos ocupa, es la composición, en porcentaje, de los diversos tamaños de agregado en una muestra. Esta proporción se suele indicar de mayor a menor tamaño, por una cifra que representa, en peso, el porcentaje parcial de cada tamaño que pasó o quedó retenido en los diferentes tamices que se usan obligatoriamente para tal medición 17. La separación de un material en diferentes fracciones, según sus tamaños, es necesaria para conocer su competencia desde una visión geotécnica. Esta clasificación comprende dos partes: por tamizado para las partículas gruesas (gravas y arenas) y el de sedimentación para la fracción fina del suelo (limos y arcillas). Por granulometría o análisis granulométrico de un agregado se entenderá todo procedimiento manual o mecánico por medio del cual se pueda separar las partículas constitutivas del agregado según tamaños, de tal manera que se puedan conocer las cantidades en peso de cada tamaño que aporta el peso total. Para separar por tamaños se utilizan mallas de diferentes aberturas, las cuales proporcionan el tamaño máximo de agregado en cada una de ellas. En la práctica los pesos de cada tamaño se expresan como porcentajes retenidos en cada malla, con respecto al total de la muestra. Estos porcentajes retenidos se calculan tanto parciales como acumulados, en cada malla, ya que con estos últimos se procede a trazar la gráfica de valores de material (granulometría).

Las curvas pueden ser representadas de varias formas, la forma más utilizada provee el uso de diagramas semilogarítmicos, con las abscisas (tamaños de mallas o de clasificación en general) en escala logarítmica y las ordenadas (porcentaje de pasante o retenido) en escala lineal. La necesidad de usar la escala logarítmica para los tamaños de clasificaciones cesaría en efecto en el caso de muestras muy homogéneas granulométricamente. Es importante en una reducción de tamaño lograr mantener las curvas granulométricas empinadas lo mayor posible.

\section{III.METODOLOGÍA}

Para el desarrollo de este trabajo se obtuvieron muestras de afloramientos, específicamente tres y de cada sitio se han obtenido 30 muestras de roca.

El primer afloramiento, denominado Cojitambo, está formado por unas andesitas, las cuales son rocas volcánicas de grano fino, son comunes, como coladas de lava en regiones orogénicas y ocasionalmente forman pequeñas intrusiones. El segundo afloramiento, denominado Racar, es un travertino, el cual se debió a la depositación 
de rocas calcáreas en una zona de procesos de sedimentación. El tercer afloramiento, denominado Tahual, está compuesto de una andesita más compacta que la del primer afloramiento. Las muestras deben ser extraídas de los diferentes afloramientos, pero evitando obtenerlas de la parte superficial de los mismos, para impedir efectos de alteración y erosión, las muestras deben ser en lo posible sanas, sin fracturas y sin signos de intemperismo.

La siguiente etapa es elaborar las probetas que generará cada muestra, en este caso vamos a obtener 60 probetas de cada sitio de estudio, estas probetas serán en pares, es decir existirá por cada muestra una probeta A y una probeta $\mathrm{B}$, las mismas que debemos tratar de elaborarlas de las mismas dimensiones, esto es $5 \mathrm{~cm} \mathrm{x} 5 \mathrm{~cm} \times$ $12 \mathrm{~cm}$. La probeta A será la que va al proceso de compresión y la probeta B la que va al proceso de trituración y clasificación (Figura 1).

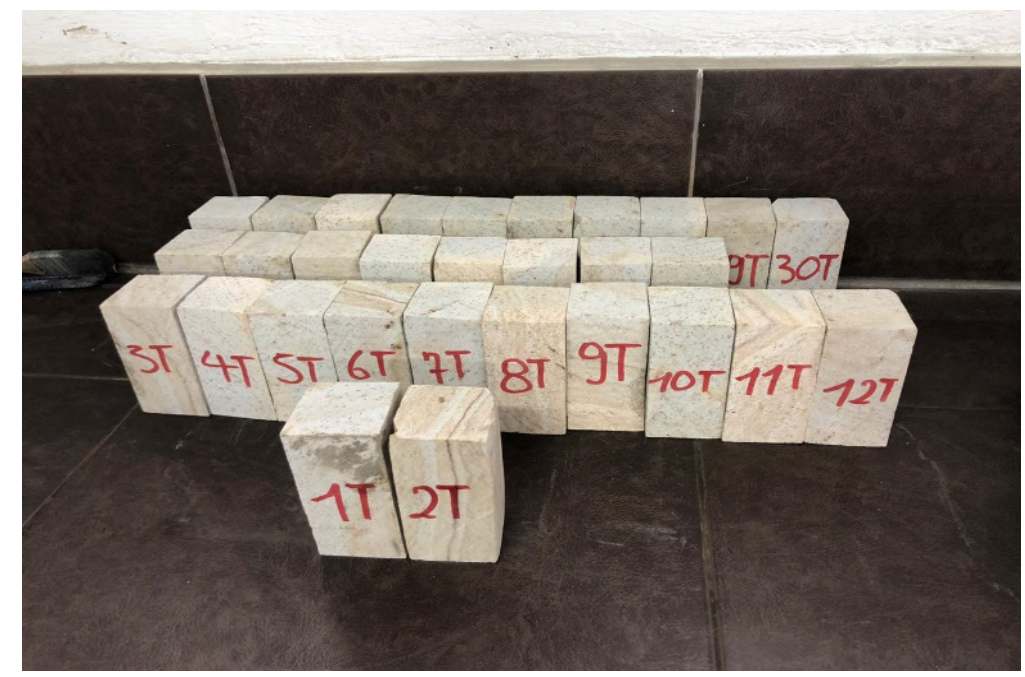

Fig. 1. Muestras o probetas de roca de Tahual

Para el afloramiento denominado Cojitambo tenemos las probetas $1 \mathrm{AC}$ y $1 \mathrm{BC}, 2 \mathrm{AC}$ y $2 \mathrm{BC}, 3 \mathrm{AC}$ y $3 \mathrm{BC} \ldots \ldots$ hasta las probetas 30AC y 30BC. Para el afloramiento Racar tenemos las probetas $1 \mathrm{AR}$ y $1 \mathrm{BR}, 2 \mathrm{AR}$ y $2 \mathrm{BR}, 3 \mathrm{AR}$ y $3 \mathrm{BR} . . . . . . . .$. hasta las probetas 30AR y 30BR. Finalmente, para el afloramiento Tahual tenemos las probetas 1AT y 1BT, 2AT y 2BT, 3AT y 3BT......... hasta las probetas 30AT y 30BT. La codificación de las muestran corresponden a la siguiente descripción: la letra A es para el ensayo de resistencia a la compresión simple, la letra B para el ensayo de trituración y clasificación, la letra C es para identificar a las probetas del afloramiento Cojitambo, la letra R para el afloramiento Racar y la letra T para el afloramiento Tahual.

Se inició el trabajo con las pruebas de resistencia a la compresión simple (probetas A). Para la ejecución de los ensayos de resistencia a la compresión se usó una prensa Humboldt, que tiene facultades para someter materiales a ensayos de tensión y compresión. La presión se logra mediante placas o mandíbulas accionadas por tornillos o sistema hidráulico. La máquina de ensayos tiene como función comprobar la resistencia de diversos tipos de materiales, para esto posee un sistema que aplica cargas controladas sobre una probeta (modelo de dimensiones preestablecidas) y mide en forma gráfica la deformación, y la carga al momento de su ruptura 18.

Paralelamente se procedió a ejecutar el proceso de trituración y clasificación (probetas B) y para la trituración se usó una trituradora de mandíbulas MEM R22.

Obtenido el material de la trituradora se lo sometió a la clasificación con los tamices $12.7 \mathrm{~mm}, 9.52 \mathrm{~mm}, 4.75$ mm y $2 \mathrm{~mm}$. El tiempo de clasificación fue de 2 minutos. Este proceso final nos proporciona las diferentes masas que son retenidas en los tamices y mediante los diagramas semilogarítmicos podemos generar las diferentes curvas granulométricas. En la figura 2, se observan las curvas del afloramiento de Cojitambo. También cabe indicar que ya se obtuvieron los diferentes valores de resistencia a la compresión simple, de las probetas para cada sitio de estudio.

\section{IV.RESULTADOS}

Obtenidas las diferentes curvas granulométricas procedemos a linealizarlas, en función de dos magnitudes x e y que se relacionan a través de una ecuación lineal, $\mathrm{y}=\mathrm{mx}+\mathrm{b}$, donde las constantes $\mathrm{b}$ (ordenada en el origen) y 
$\mathrm{m}$ (pendiente) son los valores a establecer. El método para determinar los parámetros $\mathrm{m}$ y b consiste en ingresar los datos de las curvas granulométricas en programas para que cada una de las mismas sea linealizada y de esta forma obtener dichos valores (Figura 3). La linealización se la realizó con el método de mínimos cuadrados y el objetivo principal es obtener los parámetros b y m, los cuales representan la condición que mientras menor sea el valor el producto de $b$ por $m$ entonces tendremos una mayor RCS.

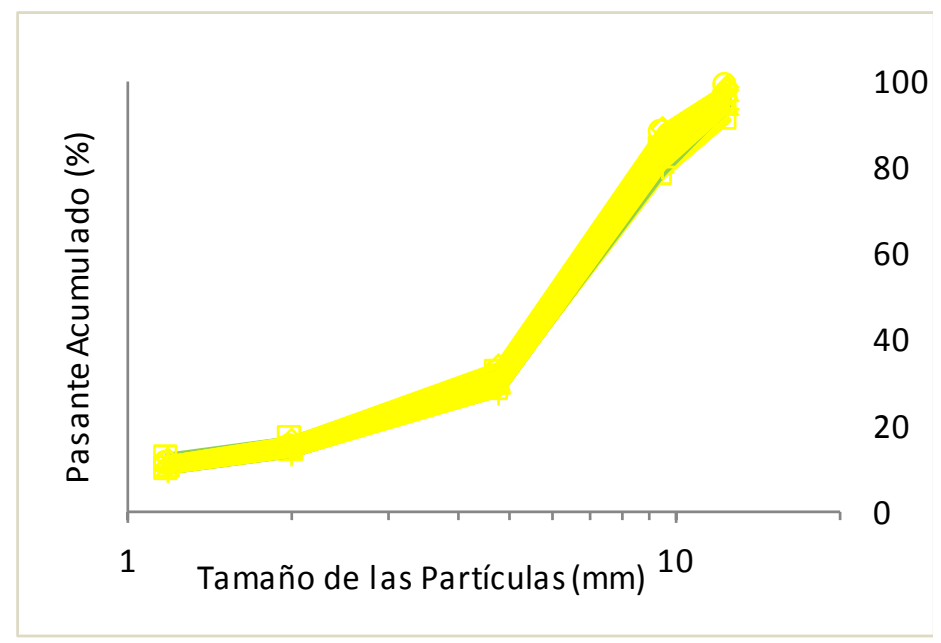

Fig. 2. Curvas granulométricas del afloramiento Cojitambo

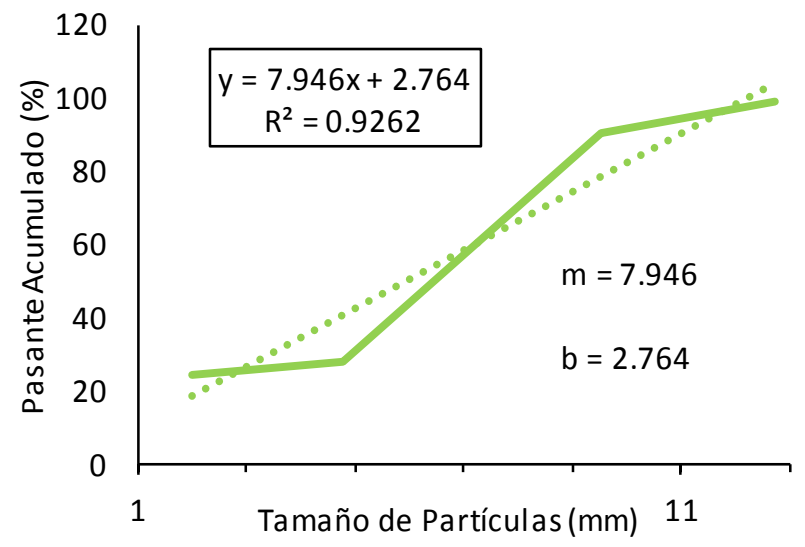

Fig. 3. Linealización de la curva granulométrica 10BR

Esta linealización se la ejecuta en las 30 curvas granulométricas de las muestras para cada afloramiento, es decir de todas las probetas B, lo que nos entrega 30 valores de pendiente (m) y 30 valores de intercepción (b). Determinados los valores se procede a identificar los respectivos pares de datos, entre la resistencia a la compresión simple de cada probeta A con la relación (bm) de cada probeta B.

Analizados los datos y conocedores de que una roca, al poseer una resistencia a la compresión más alta, al momento de un impacto, como el proporcionado por los efectos de la trituración, esta se desintegrará en pedazos de material rocoso, con más finos en su volumen final, que una roca menos resistente. Esta proposición nos conlleva a un ordenamiento de los datos, es decir, mientras más alto es el valor de la RCS de la probeta, más porcentaje de finos tendrá, en consecuencia, los valores del producto (bm) serán los más bajos. La relación existente entre la multiplicación de la intercepción y la pendiente (bm) y la resistencia a la compresión simple de las probetas, nos permitió obtener una expresión para cada sitio de estudio, es decir para cada conjunto de muestras. En el caso específico de Cojitambo tenemos la figura 4, en la cual se observa una línea de tendencia, la cual representada con la ecuación 2, nos indica la relación entre el producto de b por m y la RCS, en este caso bm se establece entre los limites -20 y 10 y el valor de la RCS entre 10 y $50 \mathrm{MPa}$. 


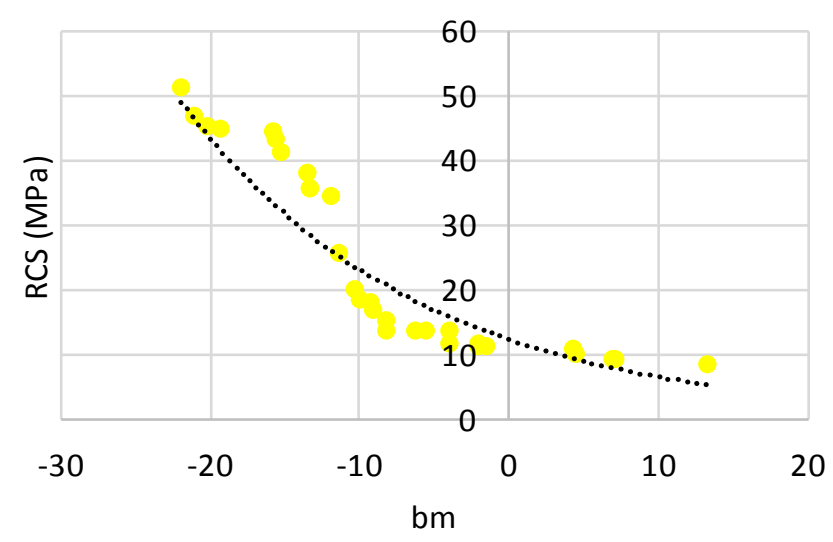

Fig. 4. RCS y la relación (bm) para Cojitambo

Podemos manifestar que la correlación entre estos valores o propiedades están sujetos a la ecuación:

$$
\operatorname{RCS}(\mathrm{MPa})=12.41 e^{-0.063 \cdot(\mathrm{bm})}
$$

Esta relación presenta un $\mathrm{R}^{2}$ igual a 0.8507 , lo cual conlleva al objetivo propuesto.

De igual forma se procede con el afloramiento Racar (Figura 5), relación que se representa con la ecuación 3, en este caso bm se establece entre los límites 10 y 100 y el valor de la RCS entre 10 y $50 \mathrm{MPa}$.

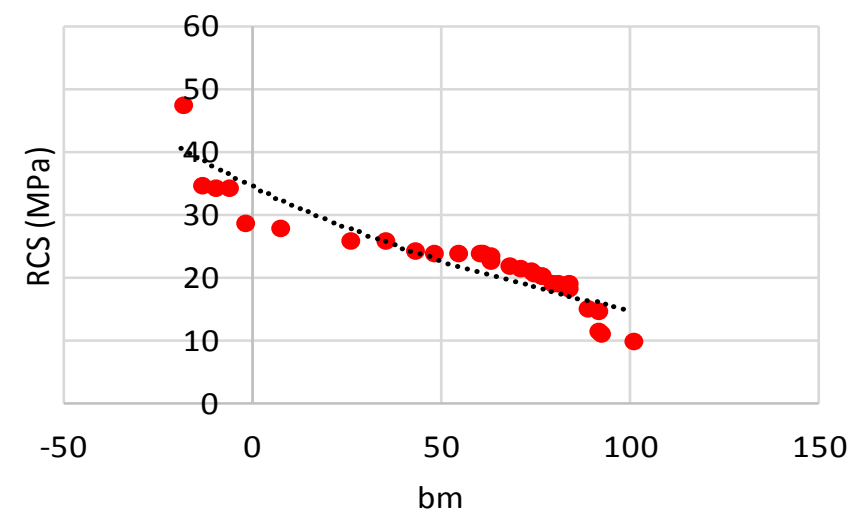

Fig. 5. RCS y la relación (bm) para Racar

La correlación entre estos valores o propiedades están sujetos a la ecuación:

$$
\operatorname{RCS}(\mathrm{MPa})=34.474 e^{-0.008 \cdot(\mathrm{bm})}
$$

Esta relación presenta un $\mathrm{R}^{2}$ igual a 0.7824 .

Finalmente, para el afloramiento Tahual (Figura 6), la relación se representa con la ecuación 4, en este caso bm se establece entre los límites -10 y 10 y el valor de la RCS entre 10 y $56 \mathrm{MPa}$. 


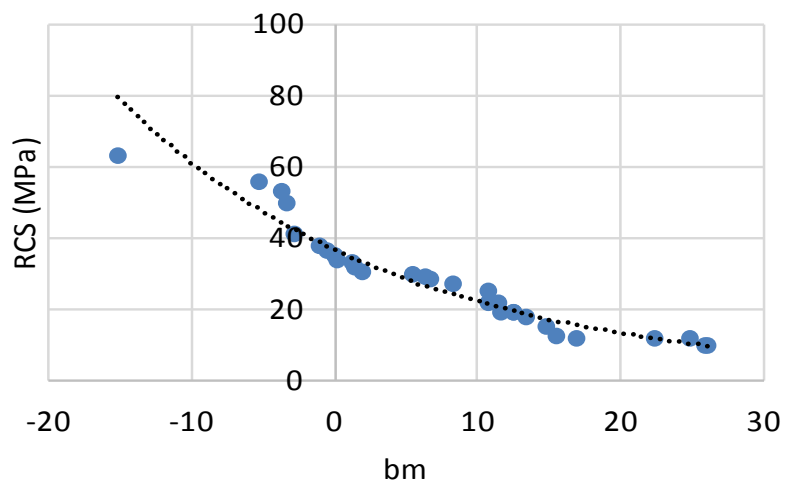

Fig. 6. RCS y la relación (bm) para Tahual

La correlación entre estos valores o propiedades están sujetos a la ecuación:

$$
\operatorname{RCS}(\mathrm{MPa})=36.612 e^{-0.051 \cdot(\mathrm{bm})}
$$

Esta relación presenta un R2 igual a 0.9497 .

Analizados los resultados iniciales, se procedió a ingresar todos los valores como un solo grupo, con lo cual obtuvimos una secuencia de graficación muy interesante, la misma se la puede observar en la figura 7. A mas de lo expuesto se obtuvo la ecuación 5, la cual permite determinar los valores de la RCS en función del parámetro bm.

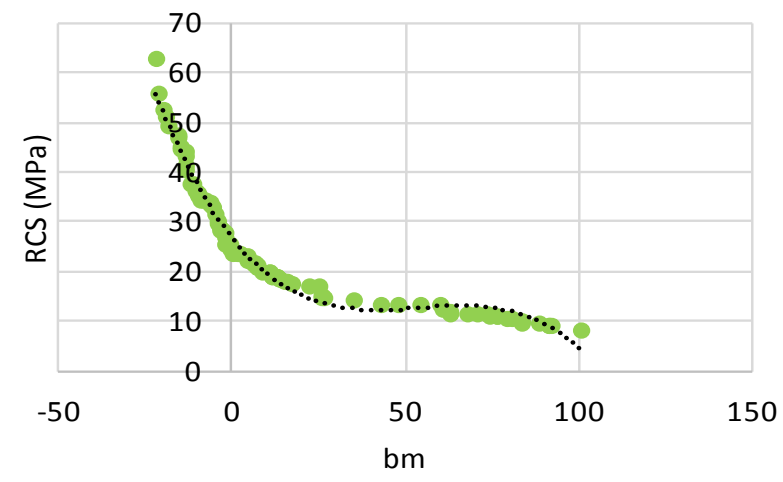

Fig. 7. RCS y la relación (bm) para todas las muestras

Esta grafica nos ha permitido encontrar una relación para los tres afloramientos y de manera bastante aproximada se la ha generado, estableciendo dominios para cada afloramiento y mediante una nueva graficación ajustada, se puede establecer cuando un valor (bm) corresponde a cada grupo de roca, a más de obtener aproximadamente el valor de la resistencia a la compresión simple. Esto se lo puede observar en la figura 8 y la ecuación 6. 


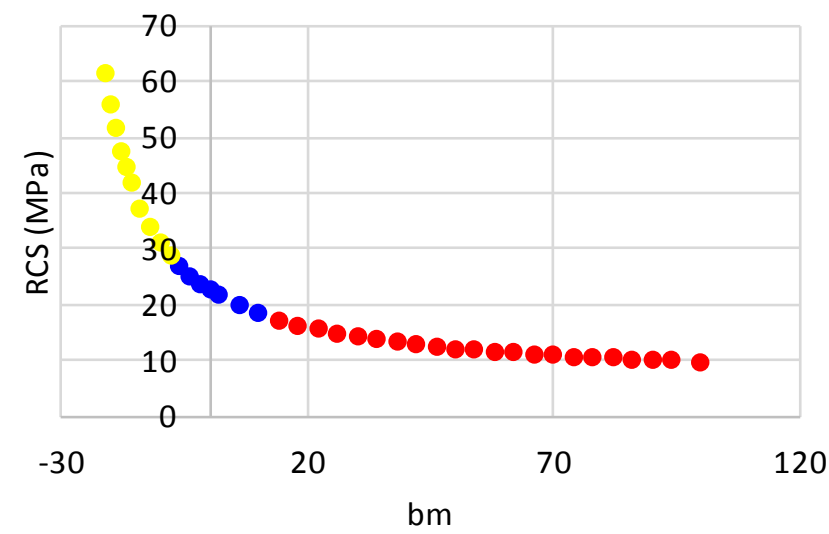

Fig. 8. Relación entre la RCS y la relación (bm)

La correlación que más se ajusta entre los datos de la figura 7 es la siguiente:

$$
R C S(M P a)=-0.0001(b m)^{3}+0.0172(b m)^{2}-0.8817 b m+26.818
$$

Esta relación presenta un $\mathrm{R}^{2}$ igual a 0.9832 .

Debido a un manejo más ágil, se propone una relación ajustada a los datos que se presentan en la figura 8, la cual es:

$$
\operatorname{RCS}(M P a)=\left(\frac{500}{(b m)+30}\right)+6
$$

Esta relación debe ser usada entre los límites:

$-20 \leq(\mathrm{bm}) \leq 100$

Estos límites se establecen, ya que el producto de bm, debe estar contenido para todos los afloramientos entre dichos valores. Además, se muestra que el error aproximado es de $\pm 5 \%$, entre las ecuaciones 5 y 6 lo que representa el usar la ecuación ajustada 6, la cual es más didáctica, en lugar de utilizar la ecuación 5.

\section{V.CONCLUSIONES}

La ejecución de pruebas de trituración y clasificación de probetas de roca, con las dimensiones descritas en este trabajo, es factible de ser ejecutadas in situ, ya que la mayor parte de empresas mineras cuentan con los equipos apropiados para dichos ensayos.

La elaboración de las curvas granulométricas, su linealización y la determinación de los parámetros (b) y (m), especificados en este trabajo, son de ejecución inmediata, mediante un software adecuado o simplemente un paquete de Microsoft Office.

Existe una correlación entre la RCS y el valor de (bm), de las diferentes muestras de rocas analizadas, lo cual nos proporciona una vía rápida, directa y económica para determinar un valor aproximado a la propiedad (RCS) de otras rocas presentes en un proyecto minero.

Para cada afloramiento se ha determinado una relación entre la RCS y el parámetro (bm), las cuales podrían permitir una aproximación de la RCS dentro de los dominios de cada una de ellas, a más de lo expuesto, en la figura 8 se establecen los valores de dominios para cada afloramiento. Para Cojitambo (bm) debe estar entre -20 y -10, para Tahual (bm) debe estar entre -10 y 10 y para Racar (bm) debe estar entre 10 y 100.

Esta propuesta nos permite determinar la RCS para rocas entre 9 a $56 \mathrm{MPa}$, es decir hasta rocas de resistencia media. Si el parámetro (bm) obtenido no está enmarcado dentro del rango alcanzado en la figura 8 , es importante 
que esos materiales sean enviados a laboratorio para la determinación de la RCS, ya que es probable que tengamos rocas de resistencia muy alta, las cuales no cumplan la propuesta mencionada.

Esta metodología es aplicable en minería, para el estudio geotécnico y comportamiento de la roca y macizo rocoso; y en base a esta determinación se podrán continuar con los estudios de uso de explosivos y evaluación de factores de seguridad, para la estabilidad, de las obras a ejecutar durante la fase de explotación de la mina o cantera.

\section{REFERENCIAS}

[1]E. P. Feijoo Calle y J. C. Padrón Suarez, «LA RESISTIVIDAD DE ROCAS Y SU RELACIÓN CON LA RESISTENCIA A COMPRESIÓN SIMPLE EN MINA», uct, vol. 24, n. ${ }^{\circ}$ 99, pp. 61-67, abr. 2020.

[2]E. P. Feijoo Calle, C. A. Flores Vera y B. A. Feijoo Guevara, "El concepto del área granulométrica y su relación con la resistencia a la compresión simple de rocas", 2019 VII Congreso Internacional de Ingeniería, Ciencias y Tecnología (IESTEC) , Panamá, 2019, págs. 52-56, doi: 10.1109/ IESTEC46403.2019.00018.

[3]F. Escolano, A. Mazariegos, Guía de reconocimiento de rocas en Ingeniería Civil. España: Editorial Garceta, Escuela Técnica Superior de Ingeniería Civil, Universidad Politécnica de Madrid, 2014.

[4]E. P. Feijoo Calle y D. M. Román Celi, «CORRELACIÓN ENTRE LA DEFORMACIÓN Y LA RESISTENCIA A LA COMPRESIÓN EN ROCAS», uct, vol. 23, n. ${ }^{\circ}$ 91, p. 6, may. 2019.

[5]L. González de Vallejo, M. Ferrer, L. Ortuño, C. Oteo, Ingeniería Geológica. España: 2004.

[6]E. P. Feijoo Calle y D. C. Iñiguez Toral, «CORTE EN ROCAS Y SU RELACIÓN CON LA RESISTENCIA A COMPRESIÓN SIMPLE». RISTI, N.o E 30, 59-67, jun. 2020.

[7]M. Galván, Mecánica de Rocas. Correlación entre la Resistencia a Carga Puntual y la Resistencia a la Compresión Simple. Colombia: Universidad del Valle, Cali, 2015.

[8]J. Alonso, J. Gómez, J. Herbert, Perforación y voladura de rocas en minería. España: Universidad Politécnica de Madrid, 2013.

[9]L. Murcia, "Procedimiento para el diseño de mallas de voladura en explotación de canteras a cielo abierto con base en la estabilidad temporal y final de los bancos de producción". Tesis de Grado, Pontificia Universidad Javeriana, Bogotá, Colombia, 2016.

[10]F. G. Blyth, M. H. de Freitas, Geología para Ingenieros. México: Cecsa, 2003.

[11]F. Arévalo, I. Cano, "Influencia de la instalación de una chancadora y faja transportadora en el incremento de producción del área de molienda de óxido de calcio Puylucana, Cajamarca". Tesis de Grado, Universidad Privada del Norte, Cajamarca, Perú, 2018.

[12]M. Colquehuanca, "Implementación de mejora al sistema de una planta de trituración de roca, para optimizar el rendimiento en el proceso de producción de agregados". Tesis de Grado, Universidad Privada del Norte, Lima, Perú, 2018.

[13]R. Calabuig, "Efecto de la adición de cal en las propiedades mecánicas y durabilidad de hormigones con altos contenidos en cenizas volantes silíceas". Tesis profesional, Piura, Universidad Nacional de Piura, Perú, 2015.

[14]D. Olguín, "Proceso de Producción de Agregados Pétreos y su Control de Calidad". Tesis de Grado, Universidad Autónoma de México, México D. F., México, 2016.

[15]C. Lara, H. Cuestas, "Determinación del wok index según la teoría de E. Bond mediante pruebas de moliendabilidad de sulfuros para el control de la etapa de molienda a nivel de laboratorio en la Facultad de Ingeniería Química U.N.C.P. Universidad Nacional del Centro del Perú". Tesis de grado, Huancayo, Perú, 2012.

[16]A. Maistri, Guía al Curso de Tratamiento de Minerales y a las Prácticas de Laboratorio.Universidad del Azuay. Ecuador: Universidad del Azuay, 1993.

[17]J. Toirac, Caracterización Granulométrica de las Plantas productoras de arena en la República Dominicana. República Dominicana, Ciencia y Sociedad, 2012.

[18]Feijoo P., Brito E., "Caracterización de la roca mediante propiedades físicas y su relación con la Resistencia a la Compresión Simple", 2020 I Congreso Internacional de Ciencia y Tecnología Morona Santiago (CICTMS), Escuela Superior Politécnica del Chimborazo, Ecuador. 


\section{RESUMEN CURRICULAR}

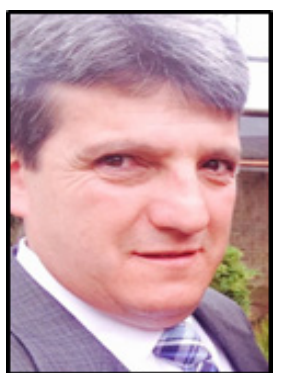

Patricio Feijoo, Ingeniero en Minas, graduado en la Universidad del Azuay (Cuenca-Ecuador), con estudios y pasantías en: Bolivia, Brasil, España, Australia en áreas de la geología, geofísica y desarrollo de actividades de explotación de minas. Se encuentra vinculado a la docencia en la Universidad del Azuay.

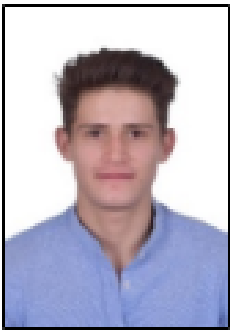

Christian Guillén, Ingeniero en Minas, graduado en la Universidad del Azuay en el 2020 (Cuenca-Ecuador), con pasantías en: Planta de beneficio "Sagrado Corazón de Jesús", Asociación Comunitaria Minera "E1 Mirador" (Ponce Enriquez) y en el Ministerio de energía y recursos no renovables zonal 6.

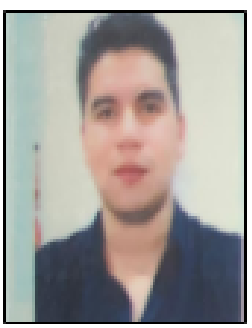

Esteban Bustamante, Ingeniero en Minas, graduado en la Universidad del Azuay en el 2020 (Cuenca-Ecuador), con pasantias en: Mineria Subterránea (GOLDEN MINNING), Planta de Beneficio y Recuperación "Corazón de Jesús", en el Ministerio de energía y recursos no renovables zonal 6 . 